\title{
WKB theory for rapid distortion of inhomogeneous turbulence
}

\author{
By S. NA Z A RE N K O ${ }^{1}$, N. K.-R. K E V L A H A N $\mathbf{N}^{2}$ \\ A N D B. D U B R U L L $\mathbf{E}^{3}$ \\ ${ }^{1}$ Mathematics Institute, University of Warwick, Coventry CV4 7AL, United Kingdom \\ ${ }^{2}$ Department of Mathematics and Statistics, McMaster University, Hamilton L8S 4K1, Canada \\ ${ }^{3}$ CNRS URA 2052, L'Orme des Merisiers, 709, 91191 Gif sur Yvette, France
}

(Received 8 April 1999)

\begin{abstract}
A WKB method is used to extend RDT (Rapid Distortion Theory) to initially inhomogeneous turbulence and unsteady mean flows. The WKB equations describe turbulence wavepackets which are transported by the mean velocity and have wavenumbers which evolve due to the mean strain. The turbulence also modifies the mean flow and generates large-scale vorticity via the averaged Reynolds stress tensor. The theory is applied to Taylor's four-roller flow in order to explain the experimentally observed reduction in the mean strain. The strain reduction occurs due to the formation of a large-scale vortex quadrupole structure from the turbulent spot confined by the four rollers. Both turbulence inhomogeneity and three-dimensionality are shown to be important for this effect. If the initially isotropic turbulence is either homogeneous in space or two-dimensional, it has no effect on the large-scale strain. Furthermore, the turbulent kinetic energy is conserved in the two-dimensional case, which has important consequences for the theory of two-dimensional turbulence. The analytical and numerical results presented here are in good qualitative agreement with experiment.
\end{abstract}

\section{Introduction}

When turbulence enters a mean flow having large velocity gradients, it experiences rapid distortion. The notion of rapid distortion refers to the fact that the interaction between the mean flow and the turbulence is much stronger than the interaction amongst the turbulent eddies, and therefore turbulence evolution may be described approximately by linear equations. Classical Rapid Distortion Theory (RDT) is based on exact solutions of these linear equations for some basic mean flows (Batchelor \& Proudman 1954; Moffatt 1965; Hunt 1973; Durbin 1981; Goldstein \& Durbin 1980). Uniform strain, uniform shear and uniform rotation are the simplest of such flows. The RDT solutions for the Reynolds stress components have had, and continue to have, a significant impact on turbulence theory. Hunt (1973) extended the RDT analysis to an important case of nonuniform strain: the distortion of initially homogeneous turbulence by steady irrotational flows around two-dimensional bluff bodies.

In the case of irrotational mean flows with nonuniform strain, the appropriate basis for RDT is given by the Cauchy's equation for the turbulent vorticity $\omega$ (Batchelor \& Proudman 1954),

$$
\boldsymbol{\omega}(\boldsymbol{x}, t)=\lambda^{-1} \boldsymbol{\omega}_{0}(\boldsymbol{a}),
$$

where $\boldsymbol{a}=\boldsymbol{a}(\boldsymbol{x}, t)$ is the initial (at $t=0$ ) coordinate of the fluid particles, $\boldsymbol{\omega}_{0}(\boldsymbol{x})=\boldsymbol{\omega}(\boldsymbol{x}, 0)$ 
is the initial vorticity and $\lambda$ is the Jacobi matrix with components

$$
\lambda_{i j}=\frac{\partial a_{i}}{\partial x_{j}}, \quad i, j=1,2,3 .
$$

Because the mean flow is initially irrotational, any mean vorticity comes from turbulent perturbations to the mean flow. Therefore, under the RDT approximation, the matrix $\lambda$ must be calculated from the solution for the initial coordinate $\boldsymbol{a}$ corresponding to the mean flow. To find $\lambda$ for stationary flows around two-dimensional bodies, Hunt expressed initial coordinate $\boldsymbol{a}$ in terms of the stream-function (constant along streamlines) and a drift function introduced by Darwin (1952) (travel time of fluid elements). Homogeneity of upstream turbulence allowed Hunt to consider the evolution of individual Fourier harmonics of initial vorticity $\hat{\omega}_{0}(\boldsymbol{q}) \exp (i \boldsymbol{q} \cdot \boldsymbol{x})$, for which (1.1) gives

$$
\hat{\boldsymbol{\omega}}(\boldsymbol{q}, \boldsymbol{x}, t)=\lambda^{-1} \hat{\boldsymbol{\omega}}_{0}(\boldsymbol{q}) \exp (i \boldsymbol{q} \cdot \boldsymbol{a}) .
$$

The solution for the vorticity may then be obtained via an integration over initial wavenumbers $\boldsymbol{q}$,

$$
\boldsymbol{\omega}(\boldsymbol{x}, t)=\int \hat{\boldsymbol{\omega}}(\boldsymbol{q}, \boldsymbol{x}, t) \mathrm{d} \boldsymbol{q} .
$$

The most important results in Hunt's approach appear for small-scale turbulence when one can introduce a local wavenumber $\boldsymbol{k}$ related to the initial wavenumber $\boldsymbol{q}$ as follows,

$$
\boldsymbol{k}=\boldsymbol{\nabla}(\boldsymbol{q} \cdot \boldsymbol{a})=\lambda^{T} \boldsymbol{q},
$$

where $\lambda^{T}$ is $\lambda$ transposed. Durbin (1981) employed Hunt's approach to consider distortion of turbulence in axisymmetric flow using Stokes function instead of the stream-function to find the Jacobi matrix. Following Hunt, Durbin (and others who studied inhomogeneous distortion of turbulence) used the Fourier transform to describe the evolution of initially homogeneous turbulence.

In many situations, however, the initial turbulence is strongly inhomogeneous. This is the case for turbulent spot experiments, and for experiments where turbulence arises in a spatially confined region of the flow. A classic experiment of this type is Taylor's four roller mill in which turbulent vortices arise in the flow confined by the four rollers. In a recent article Andreotti, Douady \& Couder (1996) describe the results of such an experiment and make the interesting observation that the mean strain is reduced by the distorted turbulence. The main goal of this paper is to modify RDT so that it applies to inhomogeneous turbulence and allows the mean flow to change in response to the small-scale turbulence.

WKB RDT has very close links with the local stability theory (also called shortwave asymptotics) developed for incompressible fluids by Lifschitz \& Hameiri (1991) and Friedlander \& Vishik (1991). In fact, some of the WKB stability calculations are identical to the classical RDT solutions, as was pointed out by Leblanc \& Cambon (1998). Lifschitz \& Hameiri (1991) noted that the WKB approach allows one to consider local perturbations to the mean flow and thus derive a universal stability condition which is independent of the boundaries, however complicated they may be. Similarly, the WKB RDT developed in the present paper allows one to consider inhomogeneous turbulence and its feedback on the mean flow locally, outside of the thin layer where the turbulence blocking by boundaries is important. The links between local stability theory and WKB RDT are discussed in $\S 2.1$. The short-wave asymptotics method of local stability theory has recently been used by Sipp \& Jacquin (1998) to study the three-dimensional elliptic instability of Taylor-Green vortices. They showed that the flow is unstable and that the fastest growing mode is the same as for unbounded elliptical flows. We consider a related 
problem from a different point of view in $\S 4$. The links between RDT and stability theory are discussed in detail in Cambon \& Scott (1999).

In $\S 2.2$ we develop a new WKB technique to extend the RDT analysis to the case of initially inhomogeneous turbulence. WKB RDT is based on Gabor transforms, rather than the Fourier transforms used in standard RDT, and thus can be applied to inhomogeneous turbulence. In addition, the theory allows the large-scale strain to be time-dependent, non-uniform and without any special symmetries (e.g. two-dimensionality or axial symmetry). Note that time-dependence is important even in flows around bluff bodies moving at constant speed because the separated flow behind the body makes the irrotational flow upstream unsteady (Cantwell \& Coles 1983). We then consider the two-dimensional limit of WKB RDT in $\S 3$. This limit is important for the theory and numerical simulation of two-dimensional turbulence.

Section 4 is devoted to the application of WKB RDT to the Taylor's four roller mill experiment of Andreotti et al. (1996). We show that turbulence inhomogeneity is crucial for the effect of strain reduction by the turbulence, and thus cannot be described using the standard RDT analysis based on Fourier transforms. Furthermore, we show that three-dimensionality of turbulence is also important and that strain reduction is absent for two-dimensional turbulence. This application shows the usefulness of WKB RDT for explaining poorly understood experimental observations. Note that the strain reduction occurs in the center of the four roller system and the boundaries are not essential for its qualitative understanding.

Finally, in $\S 5$ the results of this paper are summarized and the implications and potential applications of WKB RDT are discussed.

\section{WKB theory of the rapid distortion of turbulence}

\subsection{Links with local stability theory}

WKB RDT applies to situations in which the distortion is weakly nonuniform, so that the integral scale of turbulence $l$ is much smaller than the characteristic scale of the large-scale velocity gradient $L$,

$$
\epsilon=l / L \ll 1 .
$$

From the physical point of view, the WKB approach deals with the dynamics of vorticity wavepackets convected by the mean flow. Each wavepacket is characterized by a wavenumber $\boldsymbol{k}$ which changes due to the local strain. In general there are an infinite number of wavepackets at each point $\boldsymbol{x}$, each contributing to the turbulence spectrum which varies slowly in space. As we will see below, such wavepackets (and the turbulence spectrum) can be described in terms of the Gabor transform of vorticity.

There are close links between WKB RDT and the local stability theory developed by Lifschitz \& Hameiri (1991) and Friedlander \& Vishik (1991) to study local stability of incompressible flows with respect to localized perturbations. They considered a localized linear perturbation to a fixed solution of the incompressible Euler equations which consists of a quasi-monochromatic wave train with a slowly varying wavevector and a mean flow component which is $1 / \epsilon$ times stronger than the oscillating component. The set of equations for the wave train amplitude obtained in these papers has much in common with the equations for the turbulent component which will be derived here. There are, however, several essential differences.

First, in our approach we define the perturbations (turbulence) in such a way that their mean is zero, and we derive a separate equation for the modifications to the mean flow due to the turbulence. In principle, such modifications may accumulate slowly until they 
are as large as the original unperturbed flow. The modifications to the mean flow are as important for the present study as the evolution of turbulence itself. Secondly, our approach deals with perturbations which are not quasi-monochromatic, but contain locally an infinite wavenumber spectrum corresponding to turbulence (described in terms of the Gabor transform). Finally, our equations for turbulence have the form of a Lagrangian conservation law which allows us to write the formal solution to these equations if the mean flow trajectories are known.

In this paper, we use a technique which is similar to the one developed for the twodimensional case by Dyachenko et al. (1992) and Dubrulle \& Nazarenko (1997), and which was recently applied to the subgrid modelling of two-dimensional turbulence by Laval et al. (1998).

\subsection{Derivation of the WKB equations for turbulence}

To derive a transport equation for two-dimensional turbulence Dubrulle \& Nazarenko (1997) used Wigner's function. Here we use an alternative approach employing the Gabor transform because it leads to a shorter and more elegant derivation. The relation between the Wigner's function and Gabor (as well as wavelet) transform is well documented and can be found for example in Mallat (1998) and Farge (1992). As in the two-dimensional case, the aim is to separate the large and small scales in the original dynamical equations in such a way that the small-scale turbulence is described by a local wavenumber spectrum which varies slowly in coordinate space. This can be done by "lifting" a quantity which is conserved along the fluid-particle trajectories from coordinate space to coordinatewavenumber space (by applying Gabor transform, see below). In the two-dimensional case, the conserved quantity is the vorticity perturbation. Indeed, when the mean flow and turbulence scales are separated, the vorticity perturbation is carried unchanged by the unperturbed mean flow (to the accuracy $O\left(\epsilon^{1}\right)$ assumed in WKB theory). A similar situation arises in three dimensions for the potential vorticity vector, defined as

$$
\boldsymbol{\eta}=\lambda \boldsymbol{\omega} .
$$

Here $\boldsymbol{\omega}$ is the vorticity and $\lambda$ is a Jacobi matrix defined in (1.2). According to the Erthel's theorem, $\boldsymbol{\eta}$ satisfies

$$
\partial_{t} \boldsymbol{\eta}+\boldsymbol{u} \cdot \boldsymbol{\nabla} \boldsymbol{\eta}=0 .
$$

Equation (2.3) is equivalent to the Cauchy formula (1.1) (in fact, 1.1 is the solution to 2.3). The initial values of the potential vorticity and the vorticity coincide, $\boldsymbol{\eta}(\boldsymbol{x})=\omega(\boldsymbol{x}, 0)$.

Let us now assume that the fluid flow consists of a large-scale part corresponding to the mean flow and small-scale perturbations corresponding to turbulence,

$$
\begin{aligned}
\boldsymbol{u} & =\boldsymbol{u}_{L}(\boldsymbol{X})+\boldsymbol{u}^{\prime}(\boldsymbol{x}), \\
\boldsymbol{\omega} & =\boldsymbol{\omega}_{L}(\boldsymbol{X})+\boldsymbol{\omega}^{\prime}(\boldsymbol{x}), \\
\lambda & =\lambda_{L}(\boldsymbol{x})+\lambda^{\prime}(\boldsymbol{x}), \\
\boldsymbol{\eta} & =\boldsymbol{\eta}_{L}(\boldsymbol{X})+\boldsymbol{\eta}^{\prime}(\boldsymbol{x}),
\end{aligned}
$$

where $\boldsymbol{x}=\boldsymbol{X} / \epsilon, \quad \epsilon=l / L \ll 1$. We now linearize (2.3) with respect to the primed variables (as in RDT) and neglect $O\left(\epsilon^{2}\right)$ terms (as in WKB theory). The result is

$$
\partial_{t} \boldsymbol{\eta}^{\prime}+\boldsymbol{u}_{L} \cdot \nabla \boldsymbol{\eta}^{\prime}=0
$$

where

$$
\boldsymbol{\eta}^{\prime}=\lambda_{L} \boldsymbol{\omega}^{\prime}+\lambda^{\prime} \boldsymbol{\omega}_{L}
$$

Equation (2.6) shows that perturbations of the potential vorticity are carried unchanged 
by the mean flow. This result holds in general, not just for irrotational mean flows, however we leave rotational flows (e.g. shear flows, for which work is in progress), for separate study and consider only irrotational mean flows $\left(\boldsymbol{\omega}_{L}=0\right)$. In this case

$$
\boldsymbol{\eta}^{\prime}=\lambda_{L} \boldsymbol{\omega}^{\prime}
$$

and $\lambda^{\prime}$ drops out of the problem.

Because we need to deal with objects that are localized in both frequency and position we will use the Gabor transform representation, which is defined as

$$
\hat{\boldsymbol{\eta}}(\boldsymbol{x}, \boldsymbol{k}, t)=\int f\left(\epsilon^{*}\left|\boldsymbol{x}-\boldsymbol{x}_{0}\right|\right) e^{i \boldsymbol{k} \cdot\left(\boldsymbol{x}-\boldsymbol{x}_{0}\right)} \boldsymbol{\eta}^{\prime}\left(\boldsymbol{x}_{0}, t\right) \mathrm{d} \boldsymbol{x}_{0},
$$

where $k \sim 2 \pi / l, 1 \gg \epsilon^{*} \gg \epsilon$ and $f(x)$ is a function which decreases rapidly at infinity, e.g. $\exp \left(-x^{2}\right)$. The Gabor transform of equation (2.5) is

$$
\partial_{t} \hat{\eta}_{i}+\int f\left(\epsilon^{*}\left|\boldsymbol{x}-\boldsymbol{x}_{0}\right|\right) e^{i \boldsymbol{k} \cdot\left(\boldsymbol{x}-\boldsymbol{x}_{0}\right)} \nabla_{0} \cdot\left(\boldsymbol{u}_{L}\left(\boldsymbol{x}_{0}\right) \eta_{i}^{\prime}\left(\boldsymbol{x}_{0}\right)\right) \mathrm{d} \boldsymbol{x}_{0}=0,
$$

where $\nabla_{0}$ denotes the gradient with respect to $x_{0}$. Because of the decreasing kernel $f$, the main contribution to the integral comes from the region $\left|\boldsymbol{x}-\boldsymbol{x}_{0}\right| \sim 1 / \epsilon^{*}$. Therefore one can Taylor expand the function $\boldsymbol{u}_{L}$ since it varies significantly only over the scale $1 / \epsilon \gg 1 / \epsilon^{*}$. Neglecting the quadratic terms in this Taylor expansion, which are of order $\left(\epsilon / \epsilon^{*}\right)^{2} \ll 1$, we have

$$
\begin{aligned}
\partial_{t} \hat{\eta}_{i}+ & \int f\left(\epsilon^{*}\left|\boldsymbol{x}-\boldsymbol{x}_{0}\right|\right) e^{i \boldsymbol{k} \cdot\left(\boldsymbol{x}-\boldsymbol{x}_{0}\right)} \nabla_{0} \cdot\left(\boldsymbol{u}_{L}(\boldsymbol{x}) \eta_{i}^{\prime}\left(\boldsymbol{x}_{0}\right)\right) \mathrm{d} \boldsymbol{x}_{0}+ \\
& \int f\left(\epsilon^{*}\left|\boldsymbol{x}-\boldsymbol{x}_{0}\right|\right) e^{i \boldsymbol{k} \cdot\left(\boldsymbol{x}-\boldsymbol{x}_{0}\right)} \nabla_{0} \cdot\left[\left(\left(\boldsymbol{x}_{0}-\boldsymbol{x}\right) \cdot \boldsymbol{\nabla}\right) \boldsymbol{u}_{L}(\boldsymbol{x})\right] \eta_{i}^{\prime}\left(\boldsymbol{x}_{0}\right) \mathrm{d} \boldsymbol{x}_{0}=0
\end{aligned}
$$

Integrating by parts and changing $\nabla_{0} \rightarrow-\nabla$ in the first integral in (2.10), we see that this integral is equal to $\left(\boldsymbol{u}_{L} \cdot \boldsymbol{\nabla}\right) \hat{\eta}_{i}$. Integrating the second integral by parts and changing $\left(\boldsymbol{x}_{0}-\boldsymbol{x}\right) \rightarrow i \boldsymbol{\nabla}_{\boldsymbol{k}}$ and $\boldsymbol{\nabla}_{0} \rightarrow i \boldsymbol{k}$, the second term becomes $-\left(\boldsymbol{\nabla}\left(\boldsymbol{u}_{L} \cdot \boldsymbol{k}\right)\right) \cdot \boldsymbol{\nabla}_{\boldsymbol{k}} \hat{\eta}_{i}$. Thus, one can write the equation for $\hat{\boldsymbol{\eta}}$ as

$$
D_{t} \hat{\boldsymbol{\eta}}=0,
$$

where

$$
\begin{aligned}
D_{t} & =\partial_{t}+\dot{\boldsymbol{x}} \cdot \boldsymbol{\nabla}+\dot{\boldsymbol{k}} \cdot \boldsymbol{\nabla}_{\boldsymbol{k}} \\
\dot{\boldsymbol{x}} & =\boldsymbol{u}_{L}=\nabla_{\boldsymbol{k}} \Omega, \\
\dot{\boldsymbol{k}} & =-\nabla\left(\boldsymbol{k} \cdot \boldsymbol{u}_{L}\right)=-\nabla \Omega, \\
\Omega & =\boldsymbol{u}_{L} \cdot \boldsymbol{k} .
\end{aligned}
$$

Note that $\hat{\boldsymbol{\eta}}$ is now a vector quantity. As we have seen, the Gabor transform of the potential vorticity is carried unchanged along the rays in $(\boldsymbol{x}, \boldsymbol{k})$-space. The ray equations $(2.12)$ and (2.13) are a Hamiltonian set of equations with Hamiltonian function $\Omega$. According to (2.11), the solution for $\hat{\boldsymbol{\eta}}$ can be expressed in terms of its initial value $\hat{\boldsymbol{\eta}}(\boldsymbol{x}, \boldsymbol{k}, 0)$,

$$
\hat{\boldsymbol{\eta}}(\boldsymbol{x}, \boldsymbol{k}, t)=\hat{\boldsymbol{\eta}}(\boldsymbol{a}, \boldsymbol{q}, 0),
$$

where $\boldsymbol{a}=\boldsymbol{a}(\boldsymbol{x}, t)$ and $\boldsymbol{q}=\boldsymbol{q}(\boldsymbol{x}, \boldsymbol{k}, t)$ are respectively the initial coordinate and the initial wavenumber. 


\subsection{Properties of the rays}

In coordinate space, the turbulence wavepackets move with the mean flow (see 2.12). The solution for $\boldsymbol{x}$ in terms of initial coordinates $\boldsymbol{a}_{L}=\left(a_{1}, a_{2}, a_{3}\right)$ is given simply by the inverse of $\boldsymbol{a}_{L}(\boldsymbol{x})$.

To study wavepacket evolution in wavenumber space, it is convenient to introduce vectors $\boldsymbol{\kappa}_{i}$ corresponding to the rows of $\lambda_{L}$,

$$
\boldsymbol{\kappa}_{i}=\boldsymbol{\nabla} a_{L i}, \quad i=1,2,3 .
$$

Because the $a_{L i}$ 's are conserved along trajectories of the unperturbed mean flow, the $\boldsymbol{\kappa}_{i}$ 's satisfy

$$
\partial_{t} \boldsymbol{\kappa}_{i}+\left(\boldsymbol{u}_{L} \cdot \boldsymbol{\nabla}\right) \boldsymbol{\kappa}_{i}+\left(\boldsymbol{\kappa}_{i} \cdot \boldsymbol{\nabla}\right) \boldsymbol{u}_{L}=0, \quad i=1,2,3 .
$$

The first two terms in this equation are the Lagrangian time derivatives of $\boldsymbol{\kappa}_{i}$, and thus $\boldsymbol{\mu}_{i}(t)=\boldsymbol{\kappa}_{i}(\boldsymbol{x}(t), t)$ satisfies the equation

$$
\frac{\mathrm{d}}{\mathrm{d} t} \boldsymbol{\mu}_{i}=-\left(\boldsymbol{\mu}_{i} \cdot \boldsymbol{\nabla}\right) \boldsymbol{u}_{L}, \quad i=1,2,3 .
$$

The initial value for $a_{L i}$ is $x_{i}$, and the initial $\boldsymbol{\mu}_{i}$ is equal to the unit vector in the direction of the $i$-axis,

$$
\boldsymbol{\mu}_{i}(0)=\boldsymbol{e}_{i}, \quad i=1,2,3 .
$$

Now, if $\boldsymbol{u}_{L}$ is irrotational we can write

$$
\dot{\boldsymbol{k}}=-(\boldsymbol{k} \cdot \boldsymbol{\nabla}) \boldsymbol{u}_{L} .
$$

Thus, $\boldsymbol{\mu}_{i}(t)$ satisfies the same equation as $\boldsymbol{k}$, with initial condition (2.19). Since (2.20) is a linear equation, one can write the solution $\boldsymbol{k}(t)$ in terms of $\boldsymbol{\mu}_{i}$ and $\boldsymbol{q}=\boldsymbol{k}(0)$,

$$
\boldsymbol{k}(t)=q_{i} \boldsymbol{\mu}_{i}=\lambda_{L}^{T} \boldsymbol{q},
$$

where $\lambda_{L}^{T}$ is the transposed matrix $\lambda_{L}$. We see that the expression for $\boldsymbol{k}$ obtained via the Gabor transform does indeed coincide with the RDT definition of the local wavevector (1.5). To find the solution $\boldsymbol{k}(t)$ for a given initial value it is sufficient to know the solutions for only three initial wavenumbers, namely $\boldsymbol{e}_{1}, \boldsymbol{e}_{2}$ and $\boldsymbol{e}_{3}$. On the other hand, even these solutions are not needed for some particular mean flows for which the $\boldsymbol{a}_{L}$ 's are known so that one can calculate directly $\lambda_{L}^{T}$ to find $\boldsymbol{k}(t)$.

\subsection{Expressing the vorticity and velocity in terms of the potential vorticity}

It is not hard to see that the derivation of (2.11) does not depend on the fact that $\omega_{L}=0$. Therefore, the Gabor transform of the potential vorticity perturbations for rotational mean flows also satisfies (2.11). However, this fact is helpful only if we are able express the observables, such as velocity or vorticity, in terms of solutions of (2.11). One can do this easily for irrotational mean flows where, according to (2.7), we have

$$
\hat{\boldsymbol{\eta}}=\lambda_{L} \hat{\boldsymbol{\omega}} .
$$

Because $\left.\lambda\right|_{t=0}=1$, the initial $\hat{\eta}$ and $\hat{\omega}$ coincide, and the solution (2.15) can be written as follows,

$$
\hat{\boldsymbol{\eta}}(\boldsymbol{x}, \boldsymbol{k}, t)=\hat{\boldsymbol{\omega}}\left(\boldsymbol{a}_{L}, \boldsymbol{q}, 0\right) .
$$

From (2.22) we can find the vorticity,

$$
\hat{\boldsymbol{\omega}}=\lambda_{L}^{-1} \hat{\boldsymbol{\eta}}
$$


Let us now find $\lambda_{L}^{-1}$. In terms of $\boldsymbol{\kappa}_{i}$, equation (2.22) can be written as

$$
\hat{\boldsymbol{\eta}}=\boldsymbol{\kappa} \cdot \hat{\boldsymbol{\omega}},
$$

Multiplying the equation for $\kappa_{1}$ by $\kappa_{2}$ and subtracting the equation for $\kappa_{2}$ multiplied by $\kappa_{1}$, we have

$$
\hat{\eta}_{1} \kappa_{2}-\hat{\eta}_{2} \kappa_{1}=\left(\kappa_{1} \times \kappa_{2}\right) \times \hat{\boldsymbol{\omega}} .
$$

After vector multiplication of this equation by $\boldsymbol{\kappa}_{3}$ and using (2.25) and (2.24), we get

$$
\hat{\boldsymbol{\omega}}=\hat{\eta}_{1}\left(\boldsymbol{\kappa}_{2} \times \boldsymbol{\kappa}_{3}\right)+\hat{\eta}_{2}\left(\boldsymbol{\kappa}_{3} \times \boldsymbol{\kappa}_{1}\right)+\hat{\eta}_{3}\left(\boldsymbol{\kappa}_{1} \times \boldsymbol{\kappa}_{2}\right) .
$$

The velocity is then given by substituting (2.27) into

$$
\hat{\boldsymbol{u}}=-i \frac{(\hat{\boldsymbol{\omega}} \times \boldsymbol{k})}{|\boldsymbol{k}|^{2}} .
$$

\subsection{Effect of viscosity}

Taking into account viscosity modifies equation (2.5) as follows,

$$
\partial_{t} \boldsymbol{\eta}^{\prime}+\boldsymbol{u}_{L} \cdot \boldsymbol{\nabla} \boldsymbol{\eta}^{\prime}=\nu \boldsymbol{\Delta} \boldsymbol{\eta}^{\prime}
$$

Where $\nu$ is the viscosity. Correspondingly, (2.11) is replaced by

$$
D_{t} \hat{\boldsymbol{\eta}}=-\nu|\boldsymbol{k}|^{2} \hat{\boldsymbol{\eta}}
$$

Integrating this equation along characteristics (2.12) and (2.13) we have

$$
\hat{\boldsymbol{\eta}}(\boldsymbol{x}, \boldsymbol{k}, t)=\hat{\boldsymbol{\omega}}\left(\boldsymbol{a}_{L}, \boldsymbol{q}, 0\right) \exp (\boldsymbol{q} \Gamma \boldsymbol{q}),
$$

where

$$
\Gamma=-\nu \int_{0}^{t}\left[\lambda_{L} \lambda_{L}^{T}\right]_{\boldsymbol{x}=\boldsymbol{x}(\boldsymbol{a}, t)} \mathrm{d} t .
$$

Solutions for the vorticity and velocity can be obtained by substitution of (2.31) into (2.24) and (2.28).

\subsection{Evolution of the mean flow}

The equation for the large-scale vorticity $\boldsymbol{\omega}_{L}$ is obtained by averaging the inviscid vorticity equation over the intermediate scales (between the large and small scales). For our purposes, the most convenient procedure of averaging is

$$
\boldsymbol{\omega}_{L}=\overline{\boldsymbol{\omega}}=\int f^{2}\left(\epsilon^{*}\left|\boldsymbol{x}-\boldsymbol{x}_{0}\right|\right) \boldsymbol{\omega}\left(\boldsymbol{x}_{0}, t\right) \mathrm{d} \boldsymbol{x}_{0},
$$

where $f$ is the same function as in the Gabor transform (2.8). Then, the averaged vorticity equation is

$$
\frac{\partial \omega_{L i}}{\partial t}-S_{i j} \omega_{L j}+\epsilon_{i m n} \frac{\partial^{2} R_{j n}(\boldsymbol{x}, 0)}{\partial x_{j} \partial x_{m}}=0
$$

where $S_{i j}=1 / 2\left(\partial u_{L i} / \partial x_{j}+\partial u_{L j} / \partial x_{i}\right), \epsilon_{i j k}$ is the absolute antisymmetric tensor, and $R_{i j}(\boldsymbol{x}, 0)$ is the averaged Reynolds stress tensor at $\boldsymbol{r}=0$. Using the above definition of averaging allows us to write an expression for $R_{i j}(x, 0)$ in terms of Gabor transforms (and means that the filter function $f$ does not appear explicitly in the averages),

$$
\begin{aligned}
R_{i j}(\boldsymbol{x}, 0) & =\overline{u_{i}^{\prime}(\boldsymbol{x}, t) u_{j}^{\prime}(\boldsymbol{x}, t)} \\
& =\frac{1}{2(2 \pi)^{3}} \int \hat{u}_{i}(\boldsymbol{x}, \boldsymbol{k}, t) \hat{u}_{j}(\boldsymbol{x},-\boldsymbol{k}, t)+\hat{u}_{i}(\boldsymbol{x},-\boldsymbol{k}, t) \hat{u}_{j}(\boldsymbol{x}, \boldsymbol{k}, t) \mathrm{d} \boldsymbol{k} .
\end{aligned}
$$


$R_{i j}(\boldsymbol{x}, 0)$ represents the modification of the large-scale vorticity by the distorted turbulence. The components of this tensor describe large-scale momentum fluxes induced by turbulence and, therefore, they contain the most valuable information about turbulence. To find the averaged Reynolds stresses, one has to use the RDT solutions via the following chain of substitutions $(2.23) \rightarrow(2.27) \rightarrow(2.28) \rightarrow(2.35)$.

Note that the expression for the averaged Reynolds stresses in terms of Gabor transforms is formally identical to the standard expression for these quantities in terms of Fourier transforms. An essential difference, however, is that $R_{i j}(x, 0)$ is now a function of the spatial coordinate and, therefore, gives a nonzero contribution to the equation for the mean flow (2.34). This is because we averaged over the intermediate scales, rather than over the entire fluid volume as is common in the theory of homogeneous turbulence.

\subsection{Turbulent kinetic energy}

The Reynolds stress tensor also contains information about the density of the turbulent kinetic energy

$$
E(\boldsymbol{x}, t)=\frac{1}{2} \overline{\boldsymbol{u}^{\prime 2}}=\frac{1}{2} R_{i i}(\boldsymbol{x}, 0),
$$

where summation over the repeated index is assumed. The turbulent kinetic energy $E$ varies slowly in space on the large mean flow scale. Using substitutions $(2.23) \rightarrow(2.27)$ $\rightarrow(2.28) \rightarrow(2.35) \rightarrow(2.36)$ we have the following expression for the turbulent kinetic energy,

$$
\begin{aligned}
E(\boldsymbol{x}, t) & =\frac{1}{2(2 \pi)^{3}} \int \frac{|\hat{\boldsymbol{\omega}}|^{2}}{|\boldsymbol{k}|^{2}} \mathrm{~d} \boldsymbol{k} \\
& =\frac{1}{16 \pi^{3}} \int \frac{\left|\hat{\eta}_{1}\left(\boldsymbol{\kappa}_{2} \times \boldsymbol{\kappa}_{3}\right)+\hat{\eta}_{2}\left(\boldsymbol{\kappa}_{3} \times \boldsymbol{\kappa}_{1}\right)+\hat{\eta}_{3}\left(\boldsymbol{\kappa}_{1} \times \boldsymbol{\kappa}_{2}\right)\right|^{2}}{|\boldsymbol{k}|^{2}} \mathrm{~d} \boldsymbol{k},
\end{aligned}
$$

Because $\eta$ can be expressed in terms of the initial vorticity, as stated in (2.15), it is convenient to change the variable of integration in (2.37) from $\boldsymbol{k}$ to $\boldsymbol{q}$. Taking into account that the incompressibility in the wavenumber space means $\mathrm{d} \boldsymbol{q}=\mathrm{d} \boldsymbol{k}$ and using (2.23) and (2.21) we have

$$
E=\frac{1}{16 \pi^{3}} \int \frac{\left|\hat{\omega}_{10}\left(\boldsymbol{\kappa}_{2} \times \boldsymbol{\kappa}_{3}\right)+\hat{\omega}_{20}\left(\boldsymbol{\kappa}_{3} \times \boldsymbol{\kappa}_{1}\right)+\hat{\omega}_{30}\left(\boldsymbol{\kappa}_{1} \times \boldsymbol{\kappa}_{2}\right)\right|^{2}}{\left(q_{1} \boldsymbol{\kappa}_{1}+q_{2} \boldsymbol{\kappa}_{2}+q_{3} \boldsymbol{\kappa}_{3}\right)^{2}} \mathrm{~d} \boldsymbol{q}
$$

where $\hat{\omega}_{i 0}=\hat{\omega}_{i}(\boldsymbol{a}(\boldsymbol{x}, t), \boldsymbol{q}, 0), \quad i=1,2,3$, is the initial value of the vorticity. Similar formulae may be derived for other components of the Reynolds stress (2.35).

In general the total turbulent kinetic energy, $\int E d \boldsymbol{x}$, is not conserved. It can be transferred to, or be drawn from, the large-scale mean flow (the sum of the mean flow and turbulent energies is, of course, constant). On the other hand, according to (2.11) there is a quadratic quantity conserved by the turbulence,

$$
A=\int|\hat{\boldsymbol{\eta}}|^{2} \mathrm{~d} \boldsymbol{k} \mathrm{d} \boldsymbol{x}=\text { const. }
$$

Making the analogy with the wave action of WKB theory, we will call this invariant the turbulence action (c.f. the two-dimensional case considered in Dubrulle \& Nazarenko 1997). 


\section{Two-dimensional irrotational mean flow}

\subsection{General theory}

In many applications, the mean flow is approximately two-dimensional. The RDT analysis is much simpler for two-dimensional mean flows, and we now consider this interesting limiting case. For simplicity we drop the subscript $L$ in $\boldsymbol{u}_{L}, \lambda_{L}$ and $\boldsymbol{a}_{L}$ in this section.

Suppose that the mean flow velocity is independent of $x_{3}$,

$$
\boldsymbol{u}=\boldsymbol{u}\left(x_{1}, x_{2}, t\right) .
$$

Then, without lost of generality, we can put $u_{3}=0$, because if the mean flow is irrotational $u_{3}$ cannot depend on $x_{1}$ or $x_{2}$. In this case

$$
\begin{aligned}
& \lambda_{13}=\lambda_{23}=\lambda_{31}=\lambda_{32}=0, \\
& \lambda_{33}=1 .
\end{aligned}
$$

Because of incompressibility $\operatorname{det} \lambda=1$, and therefore

$$
\lambda_{11} \lambda_{22}-\lambda_{12} \lambda_{21}=1 \text {. }
$$

It immediately follows from (1.5) and (2.22) that

$$
\begin{aligned}
k_{3} & =q_{3}, \\
\hat{\omega}_{3}(\boldsymbol{x}, \boldsymbol{k}, t) & =\hat{\eta}_{3}(\boldsymbol{x}, \boldsymbol{k}, t)=\hat{\omega}_{3}(\boldsymbol{a}, \boldsymbol{q}, 0) .
\end{aligned}
$$

The fact that the $x_{3}$-components of the wavenumber and the vorticity are conserved is related to the equations' invariance with respect to translations along $x_{3}$-axis. Indeed, for the Hamiltonian system described by ray equations (2.12) and (2.13), the wavenumber plays the role of a momentum, and its $x_{3}$-component must be conserved. Similar conservation laws arise if the mean flow has other symmetries, e.g. axial symmetry. In the latter case, the azimuthal components of the wavenumber and vorticity are conserved along the mean flow trajectories.

We obtain the $x_{1}$ and $x_{2}$ components of vorticity using (2.27),

$$
\begin{aligned}
& \hat{\omega}_{1}=\lambda_{22} \hat{\omega}_{10}-\lambda_{12} \hat{\omega}_{20}, \\
& \hat{\omega}_{2}=-\lambda_{21} \hat{\omega}_{10}+\lambda_{11} \hat{\omega}_{20} .
\end{aligned}
$$

The expression for the energy (2.38) simplifies to

$$
E=\frac{1}{16 \pi^{3}} \int \frac{\left|\hat{\omega}_{10} \lambda_{22}-\hat{\omega}_{20} \lambda_{12}\right|^{2}+\left|-\hat{\omega}_{10} \lambda_{21}+\hat{\omega}_{20} \lambda_{11}\right|^{2}+\left|\hat{\omega}_{30}\right|^{2}}{\left(q_{1} \lambda_{11}+q_{2} \lambda_{21}\right)^{2}+\left(q_{1} \lambda_{12}+q_{2} \lambda_{22}\right)^{2}+q_{3}^{2}} \mathrm{~d} \boldsymbol{q},
$$

and expressions for the Reynolds stresses can be obtained in a similar way. In $\S 4$ we will consider a specific example for which turbulence inhomogeneity is an important factor: Taylor's four roller mill.

\subsection{Asymptotic behaviour of the energy.}

In many situations the turbulence distortion is large for large time, and we consider several such cases below. In a suitable coordinate system

$$
\begin{gathered}
\lambda_{11}=1 / \lambda_{22}=\lambda(t) \rightarrow \infty, \\
\lambda_{12}=\lambda_{21}=0 .
\end{gathered}
$$

In this case,

$$
E \approx \frac{1}{16 \pi^{3}} \int \frac{\left|\hat{\omega}_{20}\right|^{2} \lambda_{11}^{2}}{\left(q_{1} \lambda_{11}\right)^{2}+q_{3}^{2}} \mathrm{~d} \boldsymbol{q}
$$




$$
\begin{aligned}
& \approx \frac{1}{16 \pi^{3}}|\lambda| \int\left|\hat{\omega}_{20}\right|_{q_{1}=0}^{2} \mathrm{~d} q_{2} \mathrm{~d} q_{3} \int_{-\infty}^{+\infty} \frac{\mathrm{d} \tau}{\tau^{2}+q_{3}^{2}} \\
& =\frac{1}{16 \pi^{2}}|\lambda| \int \frac{\left|\hat{\omega}_{20}\right|_{q_{1}=0}^{2}}{\left|q_{3}\right|} \mathrm{d} q_{2} \mathrm{~d} q_{3} .
\end{aligned}
$$

Here we assumed that $\left|\hat{\omega}_{10}\right|_{q_{3}=0}^{2}=0$. Notice that the energy is proportional to $\lambda$ and, therefore, tends to infinity.

The simplest example of the situation considered here is the uniform strain case considered by Batchelor \& Proudman (1954). In this case $E \sim \lambda=\exp (\alpha t)$ where $\alpha$ is the strain rate. Similar behaviour is observed in more complex mean flows along streamlines passing through stagnation points (Hunt 1973).

Another example is turbulence in a mean flow generated by a line source or sink. In cylindrical coordinates $(r, \phi)$ this flow is given by

$$
u_{r}=\frac{\beta}{r}, \quad u_{\phi}=0 .
$$

Because the motion is the same on any streamline in this case, we shall consider only one of them, namely the $x_{1}$-axis. In this case $\lambda_{12}=\lambda_{21}=0$ and

$$
\lambda_{11}=1 / \lambda_{22}=x_{1} / a_{1}
$$

where

is the initial $x_{1}$-coordinate.

$$
a_{1}=-\sqrt{x_{1}^{2}-2 \beta t}
$$

In the case of a source $(\beta>0)$, we have $\lambda_{11} \gg 1$ for $x_{1} \gg a_{1}$ (i.e. $\beta t \gg a_{1}^{2}$ ). If $\beta t \gg a_{1}^{2}$, then along the $x_{1}$-axis

$$
E=\frac{1}{16 \pi^{2}} \frac{\left|x_{1}\right|}{\sqrt{x_{1}^{2}-2 \beta t}} \int \frac{\left|\hat{\omega}_{20}\right|_{q_{1}=0}^{2}}{\left|q_{3}\right|} \mathrm{d} q_{2} \mathrm{~d} q_{3}
$$

Similarly, on any other streamline

$$
E=\frac{1}{16 \pi^{2}} \frac{r}{\sqrt{r^{2}-2 \beta t}} \int \frac{\left|\hat{\omega}_{20}\right|_{q_{1}=0}^{2}}{\left|q_{3}\right|} \mathrm{d} d q_{2} \mathrm{~d} q_{3} .
$$

Note that $\hat{\omega}_{20}$ can be a function of the initial coordinates $\boldsymbol{a}=\left(a_{1}, a_{2}, a_{3}\right)$.

In the case of a sink $(\beta<0)$, we have $\lambda_{22} \gg 1$ for $x_{1} \ll a_{1}$. If $x_{1} \ll a_{1}$

$$
E=\frac{1}{16 \pi^{3}} \frac{\sqrt{x_{1}^{2}-2 \beta t}}{\left|x_{1}\right|} \int\left|\hat{\omega}_{10}\right|_{q_{2}=0}^{2}\left|q_{3}\right| \mathrm{d} q_{1} \mathrm{~d} q_{3}
$$

on the $x_{1}$-axis and

$$
E=\frac{1}{16 \pi^{2}} \frac{\sqrt{r^{2}-2 \beta t}}{r} \int \frac{\left|\hat{\omega}_{10}\right|_{q_{2}=0}^{2}}{\left|q_{3}\right|} \mathrm{d} q_{1} \mathrm{~d} q_{3},
$$

on any other streamline. Note that the trajectory of all fluid elements reaches $r=0$ in a finite time, $t=-a_{1}^{2} / 2 \beta$. Therefore, it takes a finite time for the energy to become infinite. It is interesting that the applicability condition of the WKB approach, $k \gg|\nabla u| / u$, remains valid for turbulence wave packets at any time when approaching the sink. This is because the radial component of the wavenumber grows as $k_{1} \sim q_{1} a_{1} / x_{1}$, and therefore $k u /|\nabla u| \rightarrow k_{1} x_{1} \gg 1$ if the WKB approach is valid initially, i.e. $q_{1} a_{1} \gg 1$.

If the turbulence is initially homogeneous (i.e. $\left|\hat{\omega}_{10}\right|^{2}$ independent of $\boldsymbol{x}$ ) then the energy 
grows as $\sqrt{t}$ at each point in space and as $1 / r$ near the sink. On the other hand, for $r^{2} \gg-\beta t$ the turbulence remains uniform.

\subsection{Two-dimensional turbulence in a two-dimensional mean flow}

Suppose now that the turbulent velocity is also independent of $x_{3}$,

$$
\boldsymbol{u}^{\prime}=\boldsymbol{u}^{\prime}\left(x_{1}, x_{2}, t\right) \text {. }
$$

(we allow $u_{3}^{\prime}$ to be finite). This limit is important for applications and for computations of two-dimensional turbulence. The decay of two-dimensional turbulence leads to the formation of large energetic vortices with radii of the order of the size of the fluid volume. These vortices control the dynamics of the small scales and, in particular, they control the dynamics of subgrid scales. Thus, one can linearize the small-scale equations with respect to the background mean flow generated by the large-scale vortices (as in RDT), and this leads to a great simplification in the modelling of subgrid scales in two-dimensional fluids. Applications to two-dimensional turbulence is beyond the scope of the present paper and will be reported separately (Nazarenko \& Laval 1997; Laval et al. 1998).

In this purely two-dimensional case, there is no turbulence at finite $x_{3}$-wavenumbers,

$$
\begin{aligned}
& \hat{\boldsymbol{u}}(\boldsymbol{x}, \boldsymbol{k}, t)=2 \pi \tilde{\boldsymbol{u}}\left(x_{1}, x_{2}, k_{1}, k_{2}, t\right) \delta\left(k_{3}\right), \\
& \hat{\boldsymbol{\omega}}(\boldsymbol{x}, \boldsymbol{k}, t)=2 \pi \tilde{\boldsymbol{\omega}}\left(x_{1}, x_{2}, k_{1}, k_{2}, t\right) \delta\left(k_{3}\right) .
\end{aligned}
$$

The components of $\tilde{\boldsymbol{u}}$ and $\tilde{\boldsymbol{\omega}}$ are related as follows,

$$
\begin{aligned}
& \tilde{\omega}_{1}=i k_{2} \tilde{u}_{3}, \\
& \tilde{\omega}_{2}=-i k_{1} \tilde{u}_{3}, \\
& \tilde{\omega}_{3}=i\left(k_{1} \tilde{u}_{2}-k_{2} \tilde{u}_{1}\right) .
\end{aligned}
$$

One can see that in this case the $x_{3}$ component of velocity is conserved,

$$
\tilde{u}_{3}(\boldsymbol{x}, \boldsymbol{k}, t)=i \tilde{\eta}_{2} / q_{1}=-i \tilde{\eta}_{1} / q_{2}=\tilde{u}_{3}(\boldsymbol{a}, \boldsymbol{q}, 0) .
$$

The energy can be expressed as follows

$$
E=\frac{1}{8 \pi^{2}} \int|\tilde{u}|^{2} \mathrm{~d} \boldsymbol{q}=\frac{1}{8 \pi^{2}} \int\left(\left|\tilde{u}_{30}\right|^{2}+\frac{\left|\tilde{\omega}_{30}\right|^{2}}{\left(q_{1} \lambda_{11}+q_{2} \lambda_{21}\right)^{2}+\left(q_{1} \lambda_{12}+q_{2} \lambda_{22}\right)^{2}}\right) \mathrm{d} \boldsymbol{q} .
$$

Note that the first part of this integral is conserved along the fluid trajectories. To find the second part, $I_{2}$, we change variables $q_{1}, q_{2} \rightarrow q=\sqrt{q_{1}^{2}+q_{2}^{2}}, \tau=q_{2} / q_{1}$, and integrate over $\tau$. Taking into account the fact that $\operatorname{det} \lambda=1$, we have

$$
I_{2}=\frac{A}{8 \pi^{2}} \int_{0}^{\infty} \frac{\mathrm{d} q}{q} \int_{-\infty}^{\infty} \frac{\left|\tilde{\omega}_{30}\right|^{2}}{(A \tau+B)^{2}+1} \mathrm{~d} \tau,
$$

where

$$
\begin{aligned}
& A=\lambda_{21}^{2}+\lambda_{22}^{2}, \\
& B=\left(\lambda_{11} \lambda_{21}+\lambda_{12} \lambda_{22}\right) .
\end{aligned}
$$

If the initial turbulence energy spectrum is isotropic, $\left|\tilde{\omega}_{30}\right|^{2}$ is independent of $\tau$ and

$$
I_{2}=\frac{1}{8 \pi} \int_{0}^{\infty} \frac{\left|\tilde{\omega}_{30}\right|^{2}}{q} \mathrm{~d} q .
$$

In this case $I_{2}$ is conserved along the fluid trajectories, as is the energy

$$
\left(\partial_{t}+\boldsymbol{u} \cdot \nabla\right) E=0
$$


The conservation of turbulent kinetic energy in two-dimensional turbulence was obtained previously in the special case of turbulence in a vortex-dipole flow by Dubrulle \& Nazarenko (1997). One can calculate the Reynolds stresses similarly,

$$
\begin{aligned}
R_{11} & =\frac{1}{4 \pi^{2}} \int \frac{k_{2}^{2}}{k^{4}}\left|\tilde{\omega}_{30}(\boldsymbol{a}, \boldsymbol{q})\right|^{2} \mathrm{~d} \boldsymbol{q}, \\
R_{12}=R_{21} & =-\frac{1}{4 \pi^{2}} \int \frac{k_{1} k_{2}}{k^{4}}\left|\tilde{\omega}_{30}(\boldsymbol{a}, \boldsymbol{q})\right|^{2} \mathrm{~d} \boldsymbol{q}, \\
R_{13}=R_{31}= & \frac{1}{4 \pi^{2}} \int \frac{i k_{2}}{k^{2}} \tilde{\omega}_{30}(\boldsymbol{a}, \boldsymbol{q}) \tilde{u}_{30}(\boldsymbol{a},-\boldsymbol{q}) \mathrm{d} \boldsymbol{q}, \\
R_{22}= & \frac{1}{4 \pi^{2}} \int \frac{k_{1}^{2}}{k^{4}}\left|\tilde{\omega}_{30}(\boldsymbol{a}, \boldsymbol{q})\right|^{2} \mathrm{~d} \boldsymbol{q}, \\
R_{23}=R_{32}= & -\frac{1}{4 \pi^{2}} \int \frac{i k_{1}}{k^{2}} \tilde{\omega}_{30}(\boldsymbol{a}, \boldsymbol{q}) \tilde{u}_{30}(\boldsymbol{a},-\boldsymbol{q}) \mathrm{d} \boldsymbol{q}, \\
R_{33}= & \frac{1}{4 \pi^{2}} \int\left|\tilde{u}_{30}(\boldsymbol{a}, \boldsymbol{q})\right|^{2} \mathrm{~d} \boldsymbol{q},
\end{aligned}
$$

where $k_{1}=q_{1} \lambda_{11}+q_{2} \lambda_{21}$ and $k_{2}=q_{1} \lambda_{12}+q_{2} \lambda_{22}$.

\subsection{Turbulence-induced force on the mean flow.}

For initially isotropic turbulence, $\tilde{u}_{30}(\boldsymbol{a}, \boldsymbol{q}) \equiv \tilde{u}_{30}(\boldsymbol{a}, q)$ and $\tilde{\omega}_{30}(\boldsymbol{a}, \boldsymbol{q}) \equiv \tilde{\omega}_{30}(\boldsymbol{a}, q)$, and we have

$$
\begin{aligned}
R_{11}=R_{22} & =2 I_{2}=\frac{1}{4 \pi} \int_{0}^{\infty} \frac{\left|\tilde{\omega}_{30}(\boldsymbol{a}, q)\right|^{2}}{q} \mathrm{~d} q, \\
R_{12}= & R_{21}=R_{13}=R_{31}=R_{23}=R_{32}=0, \\
R_{33} & =\frac{1}{2 \pi} \int\left|\tilde{u}_{30}(\boldsymbol{a}, q)\right|^{2} q \mathrm{~d} q .
\end{aligned}
$$

All off-diagonal components of the Reynolds stress tensor are equal to zero, and all diagonal components are conserved along the trajectories of the fluid elements. Recall that the averaged Reynolds stresses determine the feedback of small-scale turbulence on the mean flow dynamics. Thus, for initially isotropic two-dimensional turbulence this feedback has the form of a pressure (diagonal elements $R_{i i}>0$ ), whereas the turbulent viscosity is zero $\left(R_{i j}=0\right.$ for $\left.i \neq j\right)$. Note that the turbulence-induced pressure is conserved along the trajectories. Thus, the turbulence-induced pressure remains homogeneous in the case of initially homogeneous turbulence and the force produced by such a pressure on the mean flow is zero. In particular, two-dimensional isotropic homogeneous turbulence does not produce any drag on a two-dimensional obstacle embedded into the mean flow. This is a RDT generalization of d'Alembert's paradox. On the other hand, initially inhomogeneous turbulence does produce a pressure force on the mean flow. For example, a turbulent spot generated upstream of a two-dimensional body produces a positive drag force on this body during the body's entry into the spot and a negative drag when the body is leaving the turbulent region. This is because the pressure gradients produced by turbulence are stronger in front of the body due (to the stagnation point), and the direction of the pressure gradients in front of the body determines the direction of the net force.

Thus, taking into account the inhomogeneity of initially isotropic two-dimensional turbulence is important for describing the effect of turbulence on the mean flow dynamics. In the case of initially anisotropic turbulence both diagonal and off-diagonal Reynolds stresses will be finite. 


\section{Taylor's four roller mill experiment}

\subsection{Experimental results}

In a recent article Andreotti et al. (1996) describe the results of an experiment exploring the relationship between vorticity and stretching. They used a classic experimental setup, Taylor's four roller mill, to investigate the stability of a region of pure strain (a threedimensional hyperbolic straining flow in the $x_{1}-x_{2}$ plane with a linear stagnation line in the $x_{3}$-direction). The flow was found to be unstable and to produce a periodic pattern of alternate sign vortices aligned in the direction of stretching $\left(x_{1}\right)$. The most interesting observation, however, was that the amplified vortices (a sort of turbulent flow) have a negative feedback effect on the applied strain: the velocity gradient of the applied strain is weakened by the large-scale flow induced by the strained vortices. This observation is difficult to interpret using the standard RDT theory (Hunt 1973; Hunt \& Carruthers 1990), because RDT assumes that turbulence is initially homogeneous.

Andreotti et al. (1996) pointed out that because most analytical models assume that the strain is an imposed field these theories are unable to explain the experimental observations. They proposed a qualitative explanation of the effect in terms of a twodimensionalization of the vortex cores. As the vortices are amplified by stretching, their cores become increasingly intense and thus tend to oppose any external variations of velocity. Thus the velocity gradients associated with the applied strain are diminished. This explanation seems reasonable, but it is only qualitative and gives little insight into the precise interaction between the imposed strain and the amplified turbulent flow.

Our aim is to supply a detailed theoretical explanation of the negative feedback of the strained turbulence on the applied strain using the WKB RDT developed in this paper. This interaction is interesting because it is an example of a self-regulating process: the turbulence is stretched only up to a certain level, at which point it generates a large-scale flow which counteracts the applied strain and thus stabilize the interaction. Note that under inviscid linear theory the turbulence kinetic energy increases exponentially until the linear theory fails (see Kevlahan \& Hunt 1997). In order to explain this experimental observation we will make use of two observations. First, the four rollers confine the flow and thus the turbulence is necessarily inhomogeneous. Secondly, the large-scale flow is not imposed, and therefore must be allowed to vary in time and space. These two observations demonstrate that standard RDT is not sufficiently general to explain this observation. Of course, since WKB RDT it is a linear theory it is only valid for finite times, but this should be sufficient to produce the observed effect, at least qualitatively.

In this section, we present analytical and numerical results for the Reynolds stresses and mean flow evolution obtained using WKB RDT applied to the four roller experiment. These results show precisely the negative feedback effect observed in the experiments of Andreotti et al. (1996). Interestingly, the turbulence organizes itself on a large-scale separating positive and negative vorticity in order to produce a quadrupole vortex structure whose strain counteracts the applied strain. We also compare our results with the qualitative physical interpretation proposed by Andreotti et al. (1996).

\subsection{Assumptions and geometry}

The initial geometry of the flow we consider here is shown in figure 1 (in the threedimensional case the turbulence also depends on the direction $x_{3}$ perpendicular to $x_{1}$ and $x_{2}$ ). Note that we do not include the rollers themselves (there are no boundaries in our problem) and we consider only the uniform pure irrotational strain present initially at the centre of the Taylor four roller mill. The presence of the rollers is instead modelled by fact that the turbulence is inhomogeneous, i.e. it is confined initially to a circular 


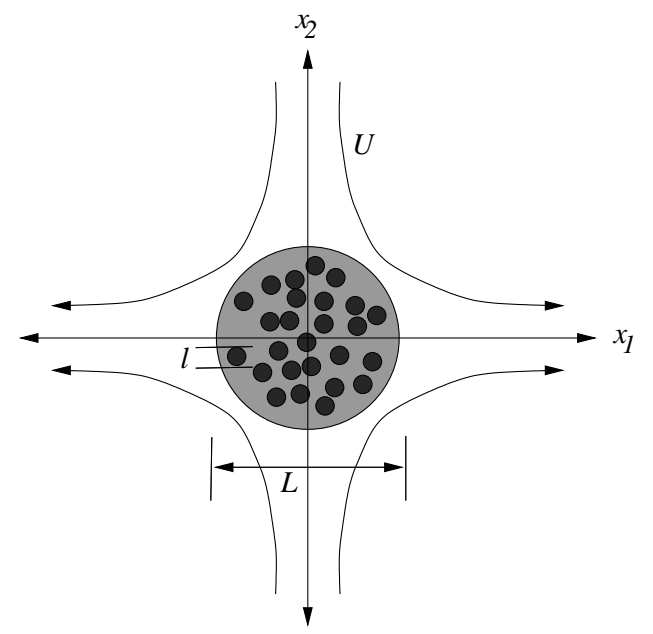

FiguRE 1. Diagram showing the geometry considered. The shaded region in the centre represents the inhomogeneous turbulence which extends over a scale $L$ much larger than the characteristic length scale of the turbulence $l$. Note that the turbulence is three-dimensional and initially isotropic while the applied irrotational strain is in the $x_{1}-x_{2}$ plane.

region with a Gaussian profile of turbulent kinetic energy. Furthermore, the characteristic length scale of the turbulence $l$ is much less than the size of the turbulent flow $L$, i.e. $l / L \ll 1$. The fact that $\epsilon=l / L \ll 1$ allows us to apply the WKB RDT to this problem. The turbulence is assumed to be homogeneous in the $x_{3}$-direction. In all calculations we assume that the initial turbulence is isotropic. This is the simplest assumption and holds for all turbulent flows at scales small enough to satisfy Kolmogorov's universal equilibrium hypothesis.

\subsection{Evolution of small-scale turbulence}

The large-scale velocity producing the applied strain in our case is initially

$$
\boldsymbol{u}_{L}=\left(S x_{1},-S x_{2}, 0\right) \text {, }
$$

which gives a positive irrotational strain $S$ in the $x_{1}$-direction and an equal negative strain $-S$ in the $x_{2}$-direction. This flow has a stagnation line in the $x_{3}$ direction. In this case the Jacobi matrix $\lambda$ becomes

$$
\lambda=\left(\begin{array}{ccc}
\exp (-S t) & 0 & 0 \\
0 & \exp (S t) & 0 \\
0 & 0 & 1
\end{array}\right) .
$$

The wavevector equation (2.13) has the following solution for the applied irrotational large-scale strain given by (4.1)

$$
\boldsymbol{k}(t)=\left(q_{1} \exp (-S t), q_{2} \exp (S t), q_{3}\right), .
$$

The spatial coordinates evolve according to (2.12), which gives

$$
\boldsymbol{x}(t)=\left(a_{1} \exp (S t), a_{2} \exp (-S t), a_{3}\right),
$$

and, using (2.15), (2.24) and (4.2) the Gabor-transformed vorticity $\hat{\boldsymbol{\omega}}$, is simply

$$
\hat{\boldsymbol{\omega}}(\boldsymbol{x}, \boldsymbol{k}, t)=\left(\hat{\omega}_{1}(\boldsymbol{a}, \boldsymbol{q}) \exp (S t), \hat{\omega}_{2}(\boldsymbol{a}, \boldsymbol{q}) \exp (-S t), \hat{\omega}_{3}(\boldsymbol{a}, \boldsymbol{q})\right) .
$$

The velocity $\hat{\boldsymbol{u}}$ may be calculated from $\hat{\boldsymbol{\omega}}$ using the formula (2.28). 


\subsection{Calculation of the perturbed large-scale flow}

Because viscosity is not important at large scales, we use the inviscid equation for the large-scale vorticity $\boldsymbol{\omega}_{L}$ given by (2.34). Our goal is to calculate the perturbed largescale vorticity $\boldsymbol{\omega}_{L}$ given the WKB RDT solutions for $R_{i j}(\boldsymbol{x}, 0)$ produced by the distorted small-scale turbulence. Because the initial large-scale vorticity is zero, (2.34) is a valid approximation for the perturbed large-scale vorticity provided the perturbations to the strain remain small compared to the initial large-scale strain. This means that we can solve for the modified large-scale vorticity field using the initial strain field (4.1).

Substituting (2.28), (4.5), (4.3) into (2.35) we can derive the following expressions for the Reynolds stress

$$
\begin{aligned}
R_{11}(\boldsymbol{x}, 0, t) & =\int \frac{E(\boldsymbol{a},|\boldsymbol{q}|)}{4 \pi|\boldsymbol{q}|^{2}|\boldsymbol{k}(t)|^{4}}\left(e^{2 S t} q_{2}^{2}\left(q_{1}^{2}+q_{2}^{2}\right)+2 q_{2}^{2} q_{3}^{2}+e^{-2 S t} q_{3}^{2}\left(q_{1}^{2}+q_{3}^{2}\right)\right) \mathrm{d} \boldsymbol{q},(4.6) \\
R_{22}(\boldsymbol{x}, 0, t) & =\int \frac{E(\boldsymbol{a},|\boldsymbol{q}|)}{4 \pi|\boldsymbol{q}|^{2}|\boldsymbol{k}(t)|^{4}}\left(e^{2 S t} q_{3}^{2}\left(q_{2}^{2}+q_{3}^{2}\right)+2 q_{1}^{2} q_{3}^{2}+e^{-2 S t} q_{1}^{2}\left(q_{1}^{2}+q_{2}^{2}\right)\right) \mathrm{d} \boldsymbol{q},(4.7) \\
R_{33}(\boldsymbol{x}, 0, t) & =\int \frac{E(\boldsymbol{a},|\boldsymbol{q}|)}{4 \pi|\boldsymbol{q}|^{2}|\boldsymbol{k}(t)|^{4}}\left(e^{2 S t} q_{1}^{2}\left(q_{1}^{2}+q_{3}^{2}\right)+2 q_{1}^{2} q_{2}^{2}+e^{-2 S t} q_{2}^{2}\left(q_{2}^{2}+q_{3}^{2}\right)\right) \mathrm{d} \boldsymbol{q},(4.8) \\
R_{i j}(\boldsymbol{x}, 0, t) & =0 \quad \text { if } i \neq j
\end{aligned}
$$

where $E(\boldsymbol{a},|\boldsymbol{q}|)$ is the initial Gabor energy spectrum and we have used the fact that for initially isotropic turbulence

$$
\hat{\omega}_{i}(\boldsymbol{a}, \boldsymbol{q}) \hat{\omega}_{j}(\boldsymbol{a},-\boldsymbol{q})=\frac{1}{4 \pi}\left(\delta_{i j}-\frac{q_{i} q_{j}}{|\boldsymbol{q}|^{2}}\right) E(\boldsymbol{a},|\boldsymbol{q}|) .
$$

Since the turbulence is confined to a circular cylinder with its axis in the $x_{3}$-direction we assume the following initial Gabor energy spectrum

$$
E(\boldsymbol{a},|\boldsymbol{q}|)=\exp \left(-\left(a_{1}^{2}+a_{2}^{2}\right) / L^{2}\right) \frac{g_{1}|\boldsymbol{q}|^{4}}{\left(g_{2}+|\boldsymbol{q}|^{2}\right)^{17 / 6}} \exp \left(-(|\boldsymbol{q}| / 10)^{2}\right),
$$

where $g_{1}=1.794$ and $g_{2}=0.5576$ are chosen to give, respectively, a total kinetic energy density $\int_{0}^{\infty} E(q) \mathrm{d} q$ of $9 / 4$ and a turbulence integral scale of $l=1$. This energy spectrum has the form $k^{4}$ at scales $k \ll 1, k^{-5 / 3}$ for $1<k<10$ and it decays exponentially for $k \gg 10$ (the dissipation scale of the turbulence). We choose $L=100$ so that $l / L=\epsilon \ll 1$, consistent with the assumptions of the WKB RDT theory.

With the Gabor energy spectrum given by (4.11) and substituting for $\boldsymbol{a}$ using (4.4) the solution for the perturbed large-scale vorticity is

$\omega_{3 L}=\frac{4 x_{1} x_{2}}{L^{4}} \int_{0}^{t} \exp \left[-\left(x_{1}^{2} \exp \left(-2 S t^{\prime}\right)+x_{2}^{2} \exp \left(2 S t^{\prime}\right)\right) / L^{2}\right]\left(R_{11}\left(\boldsymbol{x}, 0, t^{\prime}\right)-R_{22}\left(\boldsymbol{x}, 0, t^{\prime}\right)\right) \mathrm{d} t^{\prime}$.

All other large-scale vorticity components are unaffected by the turbulence. It is important to note that the spatial dependence of the perturbed large-scale vorticity is determined entirely by the initial spatial inhomogeneity of the turbulence, while the amplitude of the perturbation is given by the combined effect of the turbulence Reynolds stresses and the spatial inhomogeneity. If the turbulence were homogeneous, or if $R_{11}=R_{22}$ (which is the case for the initial undistorted turbulence) then the large scales would not be affected. Thus, we see clearly that the combined effect of spatial inhomogeneity and distorted small-scale turbulence is necessary to distort the large-scale flow. 


\subsection{Two-dimensional turbulence}

If the small-scale turbulence is two-dimensional and initially isotropic then the perturbed large-scale flow is still given by (4.12), but the Reynolds stresses are now identical, $R_{11}=R_{22}$ for all $t$ and $\boldsymbol{x}$ (see 3.39). Using this result in (4.12) we see that if the smallscale turbulence is strictly two-dimensional then the large-scale flow is not affected by the turbulence. This result is interesting because it shows that not only does the perturbation of the large-scale flow depend on the small-scale turbulence being anisotropic and inhomogeneous, but that it must also be three-dimensional. Thus, the negative feed-back effect observed by Andreotti et al. (1996) is an intrinsically three-dimensional effect that requires the small-scales to be both anisotropic and spatially inhomogeneous.

\subsection{Numerical results}

In the previous section we derived an expression for the perturbation of a large-scale straining flow due to the distorted inhomogeneous small-scale turbulence. Even though asymptotic results can be found for $S t \gg 1$ (see Kevlahan \& Hunt (1997)), these results are not relevant to our case because our approximation that $S$ is uniform is valid only close to the center and, therefore, not for very large distortions. Thus, we will concentrate on the case of small and intermediate total distortions $S t$, and solve (4.12) numerically. We consider both inviscid and viscous turbulence. According to (2.31) the only difference between inviscid and viscous turbulence is that in viscous turbulence all vorticity components decay equally at large $S t$ and large $q_{i}$ due to an extra factor

$$
\exp \left[-\frac{\nu}{2 S}\left(q_{1}^{2}(1-\exp (-2 S t))+q_{2}^{2}(\exp (2 S t)-1)+q_{3}^{2} 2 S t\right)\right] \text {. }
$$

Therefore viscosity does not change the structure of the perturbation, only its amplitude. We will see that the results are in qualitative agreement with the observations of Andreotti et al. (1996).

All calculations are made with $\epsilon=l / L=1 / 100$ and the smallest turbulence scale is $1 / 10$ the integral scale $l$ of the turbulence. The Gabor energy spectrum defined in (4.11) is used. Multi-dimensional adaptive numerical integration is used to calculate the wavenumber integrals defining $R_{11}$ and $R_{22}$, and a similar one-dimensional routine is used to calculate the perturbed large-scale vorticity via the time integral in (4.12). Only the three-dimensional case is considered since, as pointed out in $\S 3.3$, the large-scale flow is unaffected if the small-scale turbulence is two-dimensional.

Figure 2 shows the evolution of $R_{11}, R_{22} R_{33}$ and the difference $R_{11}-R_{22}$ for inviscid and viscous turbulence. The most important aspect of these figures is that $R_{11}-R_{22}$ is non-zero and negative. This means that the large-scale flow is perturbed and that the structure of the perturbation is only distorted, it does not change sign. This is equally true for the viscous case, even though the evolution of the individual vorticity components is quite different. Because the amplitude of $R_{11}-R_{22}$ is lower in the viscous case, the perturbation is weaker, although identical in spatial structure.

Figure 3 shows the large-scale vorticity field and large-scale velocity field produced by the small scale turbulence. We see that the perturbed vorticity field has a quadrupolar structure. This figure demonstrates three important results. First, the flow generated along the axes is opposite in direction and gradient to that of the initial applied strain: thus the turbulence does indeed have a negative feedback effect on the large-scale flow. Secondly, the small-scale turbulence has organized itself on a large scale separating regions of positive and negative vorticity which were initially well-mixed. Thirdly, the quadrupolar structure is a distorted and inverted version of the four rollers that create the strain in the laboratory experiment of Andreotti et al. (1996). This is a remarkable 

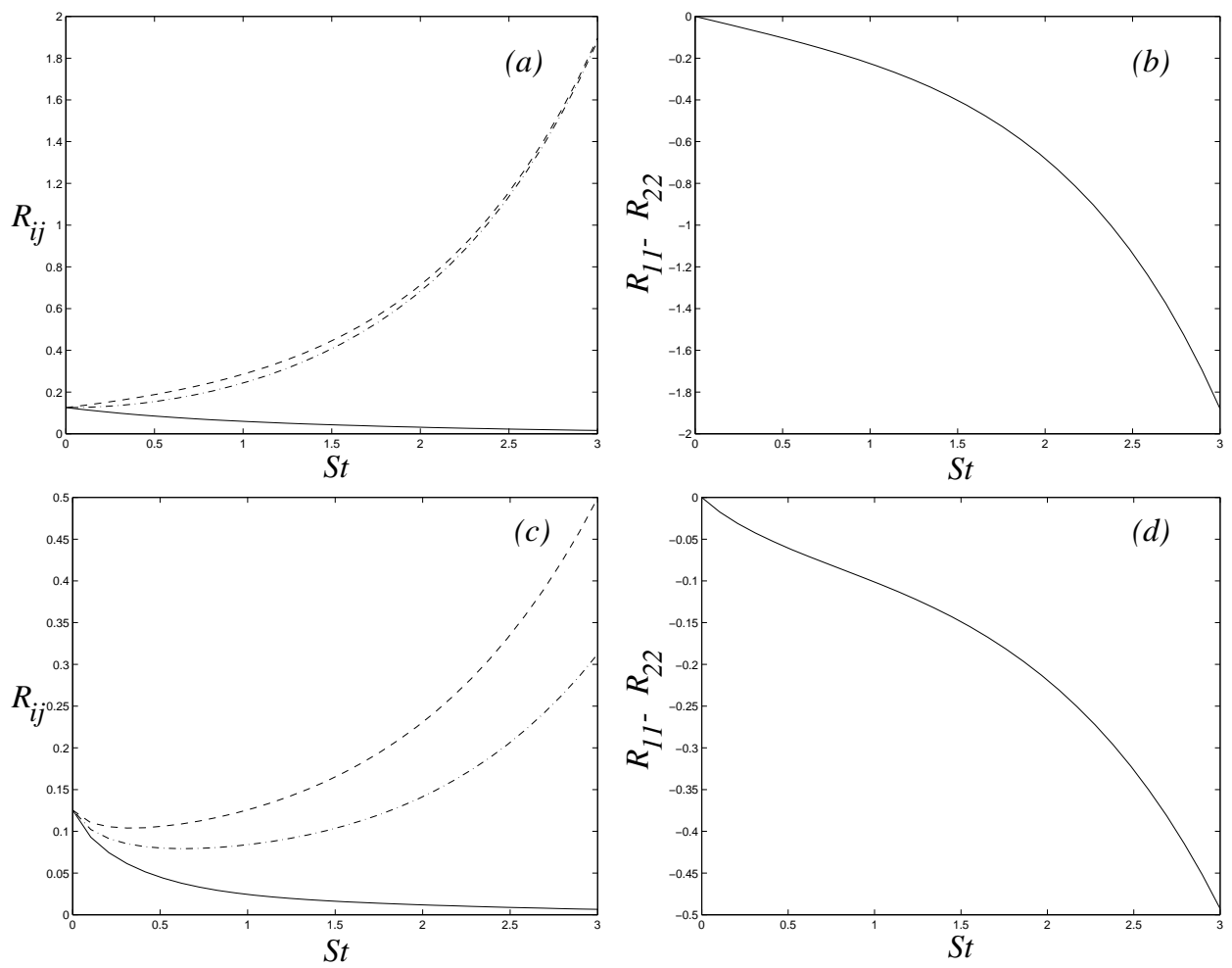

Figure 2. Evolution of Reynolds stresses for inviscid and viscous turbulence. Note that the fact that $R_{11}-R_{22}<1$ shows that the large-scale flow induced by the turbulence counteracts the applied strain. (a) Reynolds stresses in the inviscid case. - , $R_{11}$; ---, $R_{22}$; - -,$R_{33}$. (b) Evolution of $R_{11}-R_{22}$ in the inviscid case. (c) Reynolds stresses in the viscous case. $R_{11} ;---, R_{22} ;-\cdot-, R_{33}$. (d) Evolution of $R_{11}-R_{22}$ in the viscous case.

result since it shows that turbulence vorticity is not only amplified by the strain, but also re-organizes itself on the large scale of its inhomogeneity, creating a quadrupolar vortex structure that opposes the applied strain. As mentioned before, this effect requires an inhomogeneous, anisotropic (i.e. distorted) three-dimensional turbulent flow. If the $R_{11}-R_{22}$ were positive then the vortices would change sign and the perturbed flow would enhance the applied strain. Thus we see that the relative strength of $R_{11}$ and $R_{22}$ controls the type of perturbation and whether the feedback effect is negative or positive.

The development of the large-scale vorticity is shown in figure 4 . The basic quadrupolar pattern is conserved, although it is strongly distorted by the applied strain. This distortion amplifies the negative feedback which, under nonlinear theory, would eventually limit the maximum distortion (when the perturbation reaches the same order of magnitude as the applied strain). In the experiment the maximum distortion would also be limited by the boundary layers around the rollers which effectively confine the flow.

The development of the large-scale velocity profile (calculated from the vorticity fields in figure 4) are presented in figure 5. This figure shows directly that the large-scale velocity gradient induced by the small-scale turbulence opposes that of the applied strain. The gradient is rapidly amplified as the quadrupolar structure is increasingly distorted. It is 

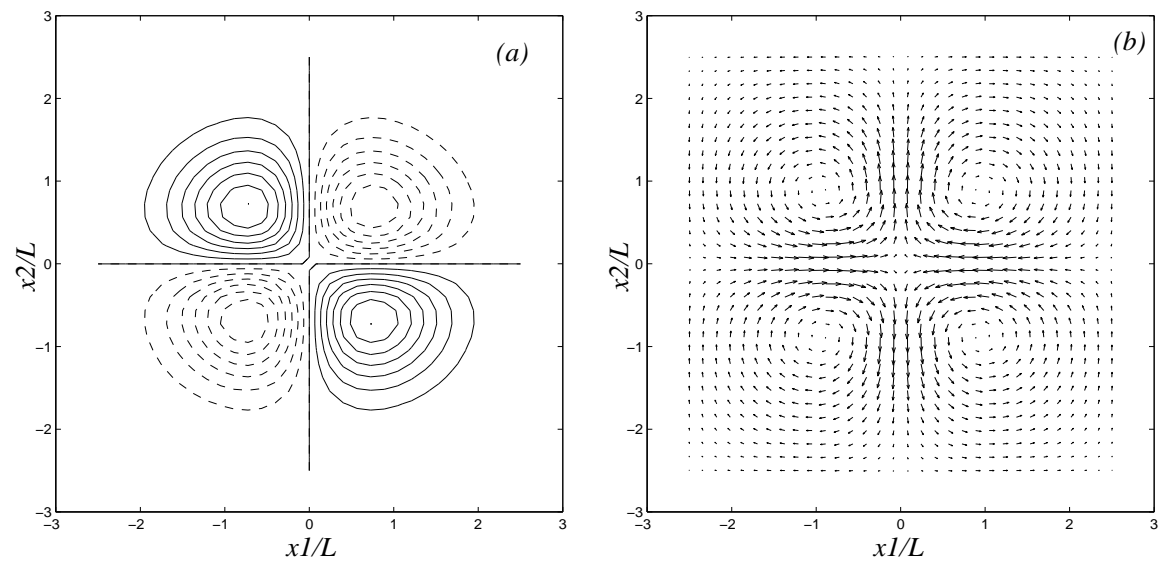

Figure 3. Contribution to the large-scale flow from distorted turbulence at $S t=0.1$ (inviscid calculation). Note that the strained turbulence has produced a large-scale flow whose velocity gradients counteract those of the applied strain. (a) Vorticity field (solid contours indicate positive vorticity dashed contours indicate negative vorticity). (b) Velocity field.
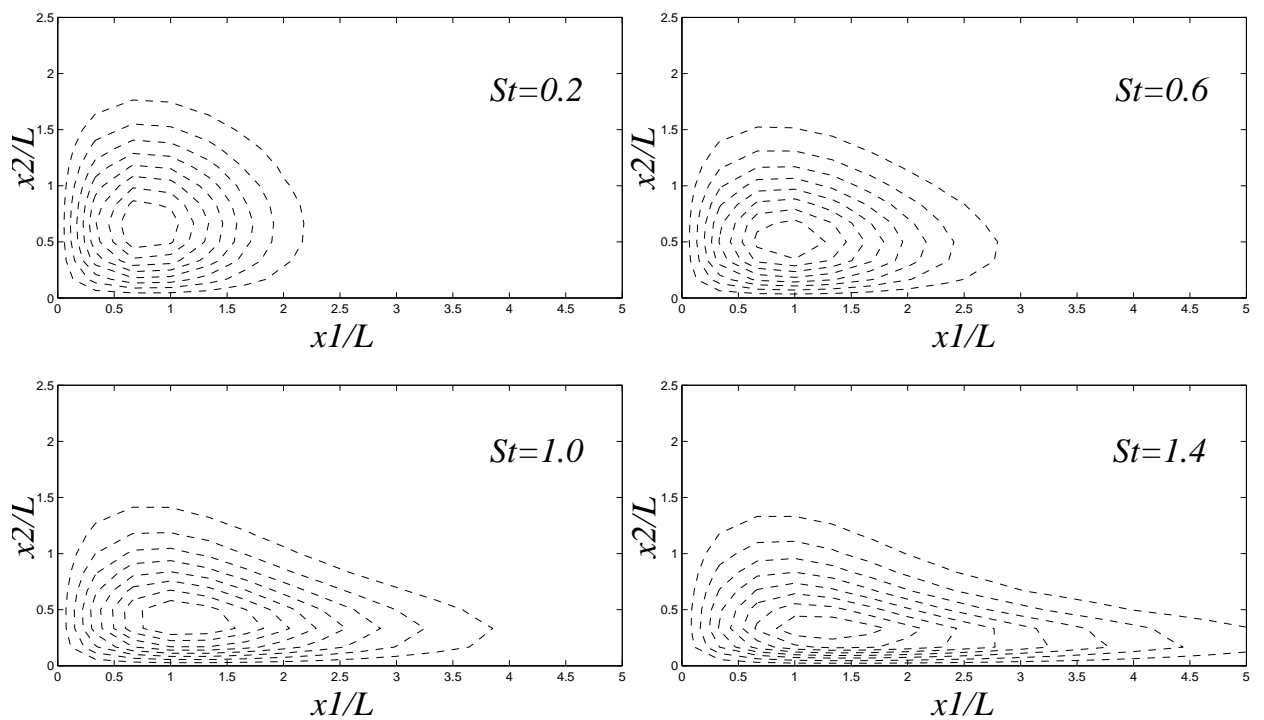

Figure 4. Development of the large-scale vorticity induced by inviscid turbulence (only the first quadrant is shown due to the quadrupole symmetry of the flow).

again remarkable that the small-scale turbulence is able to organize itself on a large-scale in order to oppose the applied velocity field.

Finally, the evolution of the maximum vorticity and the strain rate $\partial u_{1} / \partial x_{1}(0,0)$ are shown for the inviscid and viscous simulations in figure 6 . As mentioned earlier, the only difference between the inviscid and viscous cases is that the amplitude of the perturbation is greater in the former. This is because the spatial structure of the perturbation 

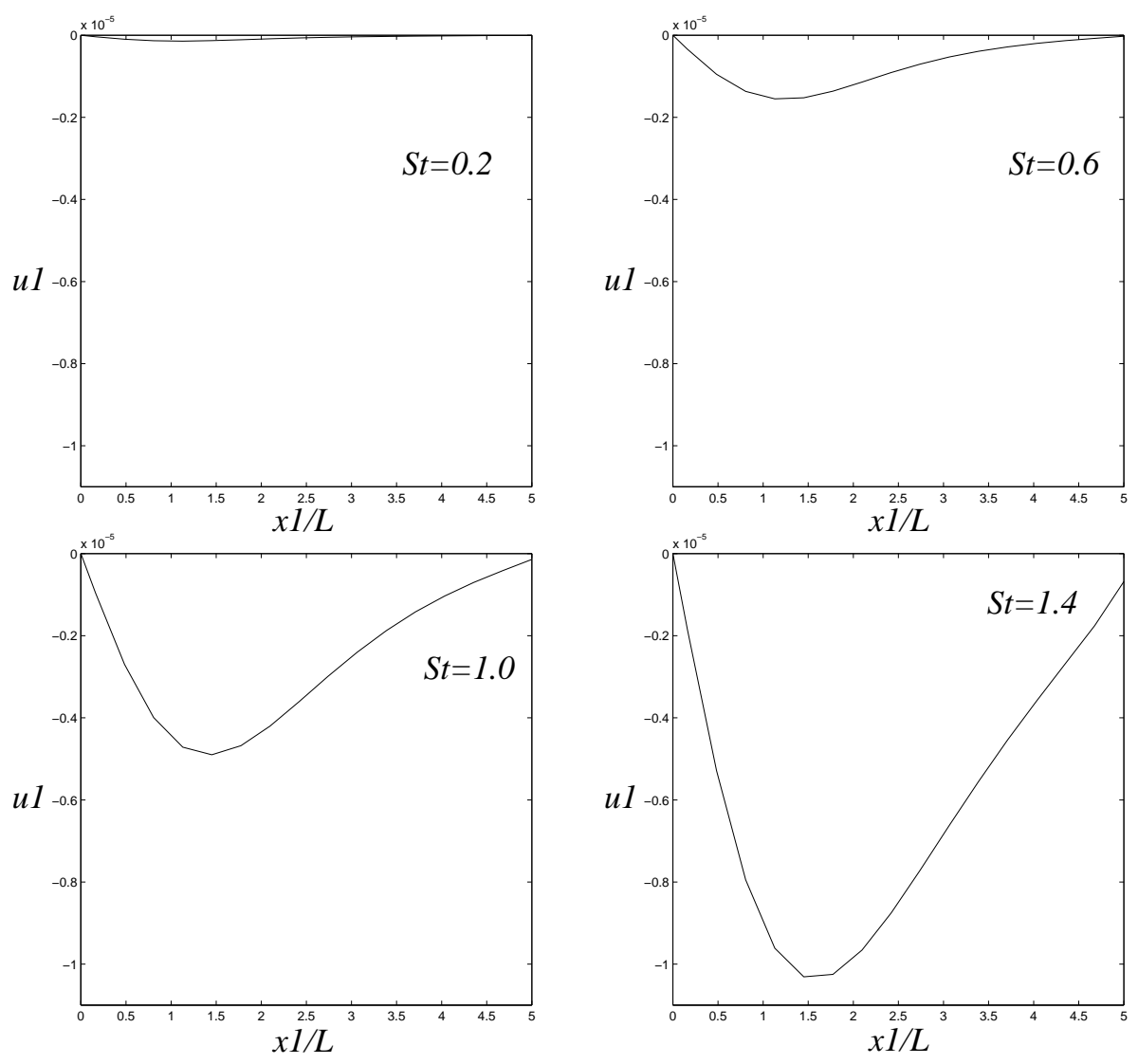

FIGURE 5 . Development of the large-scale velocity profile $u_{1}\left(x_{1}\right)$ induced by inviscid turbulence.

is determined entirely by the spatial inhomogeneity of the turbulence. The maximum vorticity and velocity gradient increase roughly exponentially in time. As expected, the growth rates are smaller in the viscous case.

\subsection{Qualitative explanation of the vortex quadrupole formation}

Formation of the large-scale vortex quadrupole can also be explained by the following simple physical argument. Consider two vortex tubes near $x_{1}=x_{2}=0$ which are initially aligned in the $x_{3}$-axis and have opposite vorticity. The total vorticity of these two tubes is zero, which corresponds to the zero mean vorticity associated with initial small-scale turbulence. If one perturbs these tubes in the $x_{3}$-direction, then the perturbation will decay in the $x_{2}$ direction and grow in the $x_{1}$ direction because of the mean strain. Thus, the vortex tubes will position themselves in the $\left(x_{1}, x_{3}\right)$ plane as shown in figure 7 . However, because of the increasing vortex-tube curvature, there will be a self-induced motion of the vortex tube segments in $x_{2}$-direction. For the vortex tube with vorticity in the positive $x_{3}$-direction, the motion will be in positive $x_{2}$-direction for $x_{1}<0$ and in negative $x_{2}$-direction for $x_{1}>0$, as shown in figure 7 . The direction of the self-induced motion in $x_{2}$-direction is reversed for the vortex tube with vorticity in the negative $x_{3}$ direction. This self-induced motion tends to separate the positive and negative vorticity and generates the quadrupole structure of $x_{3}$-vorticity in the $\left(x_{1}, x_{2}\right)$-plane. 

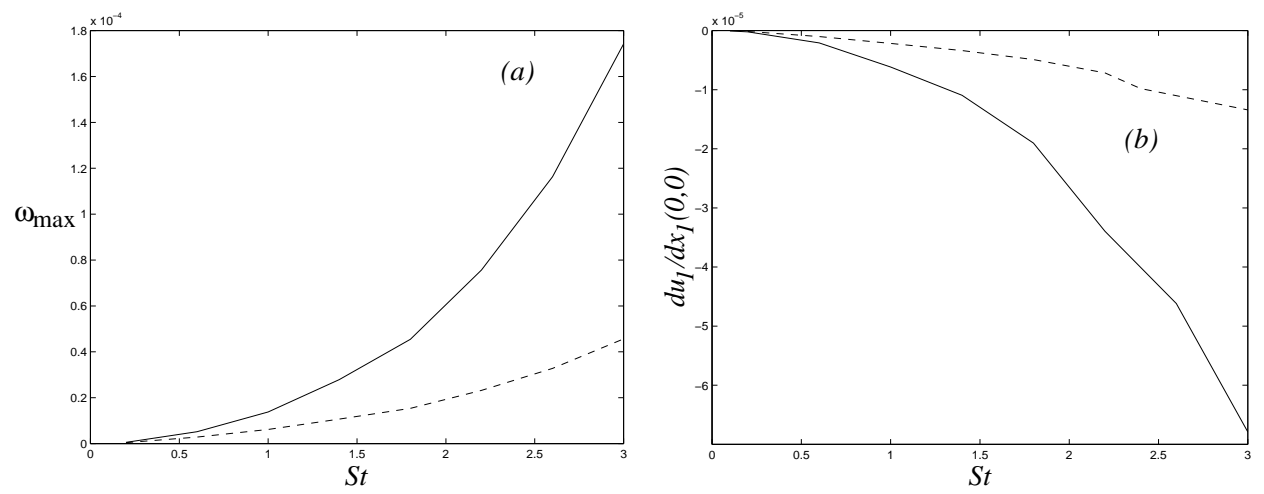

Figure 6. (a) Maximum vorticity as a function of time. - , inviscid turbulence; ---, viscous turbulence $(\nu=0.2)$. (b) Maximum velocity gradient $\partial u_{1} / \partial x_{1}(0,0)$ as a function of time. - , inviscid turbulence; ---, viscous turbulence.

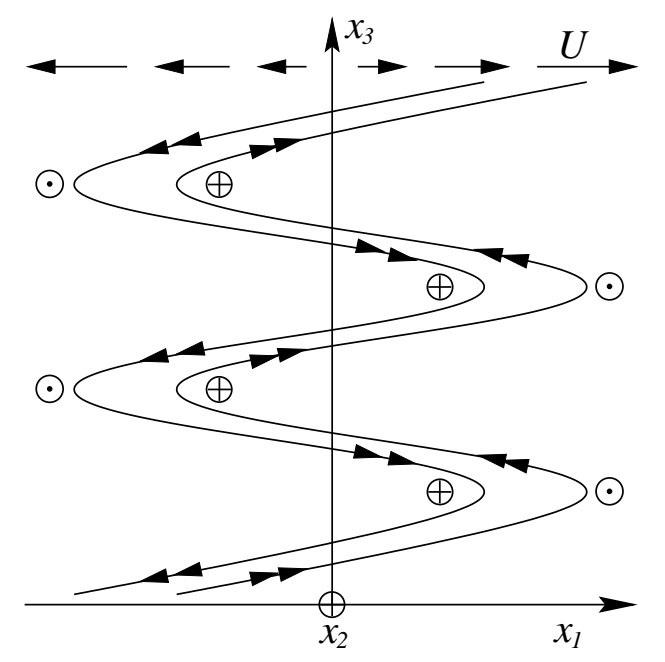

Figure 7. Physical explanation of the large-scale distortion. The curved lines are vortex tubes (vorticity direction indicated by double arrows) which were initially parallel to the $x_{3}$-axis and have been distorted by the applied strain. The small circles indicate the direction of the self-induced motion of the tubes. '.', out of the page; '+', into the page.

It is easy to see that turbulence three-dimensionality and inhomogeneity are essential for the above argument. Indeed, there would be no vortex-tube curving and self-induced motion if we did not allow the three-dimensional perturbations. On the other hand, if the density of the vortex tubes were homogeneous in the $\left(x_{1}, x_{2}\right)$-plane the net effect produced by all the vortex tubes located at different positions in the $\left(x_{1}, x_{2}\right)$-plane would be zero (for simplicity we considered an extreme inhomogeneity with vortex tubes located only at the centre).

The explanation proposed by Andreotti et al. (1996) for the negative feedback is quite different, and is based on their observation that the small-scale turbulence organizes itself into a sequence of counter-rotating vortices in the stretching direction. They suggest that once these vortices become strong enough they oppose any velocity gradients across 
their core. An analytical solution having a form of periodic distribution of vortices was suggested as a non-linear steady state for the four-mill experiment in Kerr \& Dold (1994). Our calculation does not directly involve vortices in the stretching direction (vorticity in the stretching direction is involved indirectly through the amplification of the vorticity which determines $R_{11}-R_{22}$ ), but rather the formation by the small-scale turbulence of a large-scale quadrupole in the plane of the applied strain. Our calculation gives a quantitative description of the negative feedback which reveals its necessary conditions and is in qualitative agreement with the experimental observations. Unfortunately, the authors do not include images of the flow in the plane of the applied strain, so we cannot check for the presence of the quadrupolar structure. The counter-rotating vortices observed in the experiment result from a longer-time nonlinear instability (not included in our approximation) and are not necessary for the negative feedback effect (although they may enhance it). It seems likely that the counter-rotating vortices result simply from the Kelvin-Helmholtz instability of the intense vortex sheet that forms in the $x_{1}-x_{3}$ plane during the linear straining phase.

\subsection{Comparison with the eddy-viscosity approach}

Our computation was made within the framework of scale separation, and an assumption that the small-scale turbulence is weak compared to the large-scale mean flow. Another approach exploiting scale separation was developed by Frisch and co-workers (see e.g. Frisch et al. 1987; Dubrulle \& Frisch 1991; Gama et al. 1994) who developed an asymptotic representation of eddy viscosities. They consider the opposite case: strong small-scale turbulence interacting with weak large scales. The result of such an approach is that in the presence of a strong small-scale component $u_{i}$, the Reynolds stresses $R_{i j}$ of a weak large-scale component $U_{i}$ are modified by a factor $\overline{u_{i} u_{j}}$. Because of the scale separation, this factor can be expanded as a function of $\partial_{x}^{n} U_{i}$

$$
\overline{u_{i} u_{j}}=\alpha_{i j k} U_{k}+\nu_{i j k l} \partial_{x k} U_{l}+\ldots
$$

The term proportional to $\alpha$ is the AKA term, the hydrodynamical counterpart of the alpha effect in dynamo theory; the term proportional to $\nu$ is the contribution from turbulent viscosity. In our case, the large-scale component is strong, so we cannot apply the result (4.13). However, we do have a corresponding expansion for times small with respect to the typical large-scale time scale $t \ll S^{-1}$. In this case, the Reynolds stresses $(4.6-4.9)$ are

$$
R_{i j}(t)=R_{i j}(t=0)+A_{i j} S t+\ldots,
$$

where $A_{i j}$ is a tensor which depends only on the initial spectrum of the small-scale turbulence. The first term is independent of gradients of the large-scale velocity field, but it does not contribute to vorticity generation because $R_{11}=R_{22}$ at $t=0$. This term is also absent in the eddy-viscosity approach. The second term, proportional to the gradient of the large-scale flow is a sort of "eddy-viscosity". However, it is linear in time and anisotropic, and we do not obtain any equivalent of the AKA term. It is possible, however, that a similar strain reduction effect might be obtained within the eddy-viscosity approach, because negative turbulent viscosities are known to produce large-scale instabilities leading to formation of organized multipolar structures Gama et al. (1991). Again, this would correspond to strong small scales and weak large scales, which is the inverse of the limit considered in this paper. 


\section{Conclusions}

In this paper, we have developed a WKB theory of rapid turbulence distortion using the Gabor transform. This theory provides a general description of small-scale turbulence in an irrotational mean flow. In particular, the WKB method allows the RDT analysis to be extended to initially inhomogeneous and/or anisotropic turbulence and unsteady mean flows. The WKB equations describe turbulence wavepackets which are transported by the mean velocity and have wavenumbers which evolve due to the mean strain. The energy of the turbulence wavepackets is not generally conserved: it can be transferred to or drawn from the mean flow. On the other hand, we found that the turbulence action defined in (2.39) is conserved by the turbulence.

We derived expressions for the averaged Reynolds stresses and the turbulent kinetic energy and considered the asymptotic growth of the turbulence energy under large distortions. Specific examples of mean flows produced by a source or by a sink were considered. It was shown, in particular, that the turbulent kinetic energy becomes infinite in a finite time when it is drawn into a sink.

The two-dimensional limit of the WKB equations was examined in detail. It was shown that for initially isotropic turbulence the turbulent kinetic energy is conserved along the mean flow trajectory. In this case, off-diagonal elements of the Reynolds stress tensor are equal to zero, while the diagonal ones are positive and conserved along the mean flow trajectory. In terms of the feedback of turbulence on the mean flow, this means that the turbulence produces a pressure rather than a turbulent viscosity. We established that two-dimensional turbulence does not produce any feedback on the mean flow if the turbulence is initially homogeneous (in addition to being isotropic).

Following the theoretical developments we have applied WKB RDT to the case of the interaction between large-scale plane irrotational applied strain and small-scale turbulence. The aim of this investigation was to explain the experimental observation of Andreotti et al. (1996) that the distorted small-scale turbulence generates a large-scale flow that opposes the applied strain and thus limits the maximum distortion by a negative feedback process.

In the experiment the strain is produced by four counter-rotating rollers arranged in a square; this set-up generates approximately irrotational flow in the centre of the square. We chose to approximate this flow by inhomogeneous turbulence in a uniform plane irrotational strain. (The experimental turbulence must be inhomogeneous in the plane of the strain due to the confining effect of the rollers.) The turbulence was assumed to be initially isotropic with an integral scale much less than the scale characterizing the inhomogeneity of the turbulence. By assuming that the change in the applied strain can be neglected for the purposes of calculating the distorted small-scale turbulence we were able to determine the perturbation to the large-scale flow due to the turbulence.

Using the geometry described above and the linear WKB RDT calculation we were able to produce the experimentally observed negative feedback. The small-scale turbulence re-organizes itself on the large scale of its inhomogeneity by separating positive and negative vorticity, forming a quadrupole. This quadrupole is effectively an inverted and distorted version of the four roller flow and thus opposes the velocity field of the applied strain. In order for the large-scale flow to be affected, the small-scale turbulence must be inhomogeneous, anisotropic (due to the distortion), and three-dimensional. In other words, if the initial turbulence is two-dimensional or homogeneous the large-scale flow is stationary. This result is interesting because it shows that the effect observed by Andreotti et al. (1996) is entirely three-dimensional in nature and requires that the turbulence be confined to a finite area in the plane of the applied strain. The re-organization of the 
turbulence at large-scales is also novel, and shows that the dynamics of turbulence at large-scales can be very different from those of the small scales. Although our calculation is only valid for short times, the self-stabilizing character of the interaction between large and small scales suggests that the results are valid over a longer time than might be thought a priori (for another example of the reduction of nonlinear effects in strained turbulence see Kevlahan \& Hunt 1997).

The WKB approach developed in this paper can be generalized to include the effects of compressibility, in which case an appropriate starting point for the derivation of the transport equations could be the RDT equations derived by Goldstein (1978). One can also develop a WKB approach for some special cases of rotational flows, such as nonuniform pure shear flows and nonuniform rotation. Naturally, there is one generalization which cannot be made within the WKB approach, namely the case when turbulence and the mean flow have comparable spatial scales. The effects of the finite turbulence spatial scales have been considered by Goldstein \& Durbin (1980).

In this paper we did not consider the interaction of turbulence with a wall, primarily because we did not find an application where the initial inhomogeneity of turbulence leads to new physical effects (the main wall effects for homogeneous turbulence have been described in detail by Durbin \& Hunt 1980). Note that wall effects are not needed to explain the strain reduction effect in the Taylor's four roller flow discussed in this paper.

To summarize, this paper presents the first complete analysis of the interaction between large-scale strain and small-scale inhomogeneous turbulence, and applies this new theory to the Taylor four roller mill experiment. The results have explained a previously puzzling observation: that strained small-scale turbulence can produce a negative feedback effect, reducing the gradients of the large-scale applied strain. Furthermore, the case presented here serves as an example of the striking qualitative differences between the dynamics of inhomogeneous and homogeneous turbulence. Indeed, the negative feedback effect requires that the small-scale turbulence be initially inhomogeneous and three-dimensional.

\section{REFERENCES}

Andreotti, B., Doundy, S. \& Couder, Y. 1996 About the interaction between vorticity and stretching in coherent structures. In Turbulence modeling and vortex dynamics, proceedings of a workshop held at Istanbul, Turkey, 2-6 September 1996 (ed. O. Boratav, A. Eden \& A. Erzan), pp. 92-108.

Batchelor, G. K. \& Proudman, I. 1954 The effect of rapid distortion of a fluid in turbulent motion. Q. J. Mech. Appl. Math. 7, 83.

Cambon, C. \& Scott, J. F. 1999 Linear and nonlinear models of isotropic turbulence. Ann. Rev. Fluid Mech 31, 1-53.

Cantwell, B. \& Coles, D. 1983 An experimental study of entrainment and transport in the turbulent near wake of a circular cylinder. J. Fluid Mech. 136, 321-374.

Darwin, C. G. 1952 Note on hydrodynamics. Proc. Camb. Phil. Soc. 49, 342.

Dubrulle, B. \& Frisch, U. 1991 The eddy-viscosity of parity invariant flow. Phys. Rev. A 43, 5355-5364.

Dubrulle, B. \& Nazarenko, S. 1997 Interaction of turbulence and large-scale vortices in incompressible 2D fluids. Physica D 110, 123-138.

Durbin, P. A. 1981 Distorted turbulence in axisymmetric flow. Q. J. Mech. Appl. Math. 34, 489.

Durbin, P. A. \& Hunt, J. C. R. 1980 On surface pressure fluctuations beneath turbulent flow round bluff bodies. J. Fluid. Mech. 100, 161-184. 
Dyachenko, A., Nazarenko, S. \& Zakharov, V. 1992 Wave-vortex dynamics in drift and beta-plane turbulence. Phys. Lett. A 165, 330.

FARge, M. 1992 Wavelet transforms and their applications to turbulence. Ann. Rev. Fluid Mech 24, 395-457.

Friedlander, S. \& VishiK, M. M. 1991 Instability criteria for the flow of inviscid incompressible fluid. Phys. Rev. Lett. 66, 2204-2206.

Frisch, U., She, Z. S. \& Sulem, P. L. 1987 Large-scale flow driven by the anisotropic kinetic alpha effect. Physica D 28, 382-392.

Gama, S., Frisch, U. \& Scholl, H. 1991 The 2-D Navier-Stokes equations with a large scale instability of the Kuramoto-Sivashinsky type: numerical exploration on the connection machine. J. Sci. Comp. 6, 425-452.

Gama, S., Vergassola, M. \& Frisch, U. 1994 Negative eddy-viscosity in isotropically forced two-dimensional flow: linear and non-linear dynamics. J. Fluid Mech. 260, 95-126.

Goldstein, M. E. 1978 Unsteady vortical and entropic distortions of potential flows round arbitrary obstacles. J. Fluid Mech. 89, 433-468.

Goldstein, M. E. \& Durbin, P. A. 1980 The effect of finite turbulence spatial scale on the amplification of turbulence by a contracting stream. J. Fluid Mech. 98, 473-508.

Hunt, J. C. R. 1973 A theory of turbulent flow round two-dimensional bluff bodies. J. Fluid Mech. 61, 625-706.

Hunt, J. C. R. \& Carruthers, D. J. 1990 Rapid distortion theory and the 'problems' of turbulence. J. Fluid Mech. 212, 497-532.

Kerr, O. S. \& Dold, J. W. 1994 Periodic steady vortices in a stagnation-point flow. J. Fluid Mech. 276, 307-325.

Kevlahan, N. K.-R. \& Hunt, J. C. R. 1997 Nonlinear interactions in turbulence with strong irrotational straining. J. Fluid Mech. 337, 333-364.

Laval, J.-P., Dubrulle, B. \& Nazarenko, S. 1998 Dynamical modelling of sub-grid scales in 2D turbulence. J. Fluid. Mech. (submitted).

Leblanc, S. \& Cambon, C. 1998 Effects of the Coriolis force on the stability of Stuart's vortices. J. Fluid Mech. 356, 353-379.

Lifschitz, A. \& HAmeiri, E. 1991 Local stability conditions in fluid dynamics. Phys. Fluids $A$ 3, 2644-2651.

Mallat, S. 1998 A wavelet tour of signal processing. Academic Press.

Moffat, H. 1965 The interaction of turbulence with strong wind shear. In Proc. URSI-IUGG Coll. on Atmos. Turbulence and Radio Wave Propag., Moscow, Nauka, p. 139.

NazArenko, S. \& Laval, J.-P. 1997 Nonlocal 2D turbulence and passive scalars in Batchelor's regime. J. Fluid Mech. (submitted).

SipP, D. \& JACQUin, L. 1998 Elliptic instability in two-dimensional flattened taylor-green vortices. Phys. Fluids 10, 839-849. 